\title{
Bruno Thibault*
}

\section{L'espace de la postmémoire dans Alma (2017) de J.M.G. Le Clézio}

DOI: http://dx.doi.org/10.12775/LC.2020.023

Résumé: Peut-on véritablement se mettre dans la peau d'un autre, c'est-à-dire à la place d'un autre? Alma est un roman construit sur deux parcours opposés et deux voix entrecroisées, et curieusement sur une rencontre qui n’a pas vraiment eu lieu. Jérémie Felsen fait résonner le sanglot de l'homme blanc dans le texte: son séjour sur l'île Maurice se transforme très vite en une enquête sur l'histoire de sa famille, les plantations sucrières d'antan et la question de l'esclavage. Doublant la voix de Jérémie s'élève la voix sourde, incohérente mais incessante de Dodo, le clochard qui hante les cimetières. Cette voix dit la misère, la solitude, le racisme, le traumatisme, l'errance et l'exclusion dans l'île Maurice aujourd'hui, mais aussi en France. L'analyse détaillée des expériences de ces deux personnages nous permettra d'insister sur leur rencontre avec le réel - par-delà les clichés touristiques mais aussi sur leur rencontre avec le ciel étoilé d'où le thème de l'extase chamanique. Dans notre conclusion, nous montrerons comment le réalisme magique leclézien produit un récit indécidable.

Mots clés: postmémoire, esclavage, ciel étoilé, réalisme magique, récit indécidable

\section{The Space of Postmemory in J.M.G. Le Clézio’s Alma (2017)}

\footnotetext{
Abstract: Is it really possible to put oneself in someone's shoes, in other words entirely in someone else's place? Alma is a novel constructed on two divergent paths and two interconnected voices, and rather oddly on a meeting which never actually took place. Jérémie Felsen makes the text resonate with the sobs of the white man: his stay on the island of Mauritius is quickly transformed into an

* Professeur de littérature française à l'université de Delaware. Ses recherches portent sur les écritures romanesques de l'extrême-contemporain, en particulier sur les questions liées à l'intertextualité et à l'intermédialité, ainsi que sur la rhétorique spéculative.

E-mail: thibault@udel.edu |ORCID: 0000-0003-3666-539X.
} 
investigation of his family history, the sugar plantations of the past and the issue of slavery. A hollow voice doubles Jérémie's: the incoherent yet incessant voice of Dodo, the vagrant who haunts the cemeteries. This voice expresses the misery, the solitude, the racism and the exclusion which exist not only on the island of Mauritius today, but also in France. The detailed analysis of the experiences of these two characters will highlight their encounter with the real world - beyond the tourist clichés but also their encounter with the starry skies (i.e. the theme of shamanic ecstasy). In our conclusion, we will show how leclézian magic realism produces an ambiguous narrative.

Keywords: postmemory, slavery, stary skies, magic realism, ambigios narrative

omme l'a observé Van Kelly, il existe chez Patrick Modiano et chez Jean-Marie Gustave Le Clézio, d'œuvre en œuvre, une véritable quête du père absent. D’autre part leurs récits gravitent autour d'une mémoire collective traumatique: la shoah et l'antisémitisme chez Modiano; l'esclavage et le colonialisme chez Le Clézio. Cependant il s'agit moins pour ces deux auteurs de reconstituer des événements du passé avec un souci de cohérence et de continuité narrative, comme le ferait par exemple un historien ou un écrivain militant produisant une sorte de réquisitoire, que de marquer un deuil impossible en relevant au fil des pages les signes d'un silence ou les traces d'une disparition (Kelly 2018: 39-57).

Van Kelly observe en outre que depuis Désert, notamment dans Étoile errante, dans Le Chercheur d'or et dans La Quarantaine, Le Clézio mène une étrange expérimentation pour savoir s'il est légitime (et si oui, jusqu'à quel degré?) pour un narrateur intra-diégétique de fusionner avec l'expérience d'autres personnages, en réactualisant les conflits et les souffrances liés aux circonstances historiques qui ont forgé leur identité. Peut-on véritablement se mettre dans la peau d'un autre, c'est-à-dire à la place d'un autre? Question qui est ouverte à tous mais qui s'adresse plus particulièrement aux romanciers, dont l'imagination anime la conscience de créatures plus ou moins fictionnelles. Dans le cas de Le Clézio, il y a là quelque chose d'irrépressible: cette empathie, cette pulsion d'identification et de fusion, s'exprime non seulement dans plusieurs de ses romans mais aussi dans certains textes autobiographiques, Voyage à Rodrigues et Gens des nuages par exemple.

Le Clézio met souvent en scène un personnage malmené par les soubresauts de l'histoire, telle Esther dans Étoile errante, puis il relate une rencontre inopinée, celle d'une conscience jumelle mais rivale - ici la jeune Palestinienne nommée Nejma - que l'Histoire tient également dans ses griffes. Comme l'ont noté Jean-Philippe Imbert et Walter Putnam, ${ }^{1}$ cette rencontre se traduit par une sorte de fusion spirituelle des deux personnages mais cette fusion ne dure qu'un instant car Nejma est aussitôt emportée en camion vers un camp de réfugiés. Pourtant le visage de l'Autre ne cesse de hanter la conscience du personnage-pivot de la narration, Esther. Une certaine identification opère ici, mêlée de compassion mais aussi de culpabilité - identification qui débouche sur un rêve de solidarité et un fantasme de réciprocité, sur le vœu pieux de se retrouver un jour à nouveau ensemble, et, en attendant, par le truchement de l'écriture d'un journal intime. Esther considère en effet que Nejma, en

\footnotetext{
1 Je renvoie aux deux essais de Jean-Philippe Imbert 1997 et de Walter Putnam 1998.
} 
traçant les lettres de son nom dans son cahier noir, l'a encouragée à écrire son histoire, une "histoire d'amour et d'errance" (Le Clézio 1992: 284).

L'emploi du dialogue intérieur et imaginaire, où les voix des deux jeunes femmes semblent parfois se mêler, produit une sorte de réconciliation virtuelle très frappante dans Étoile errante. Cependant, comme le souligne Van Kelly à la suite d'autres critiques, ${ }^{2}$ il existe un certain déséquilibre narratif - "a narrative imbalance" (Kelly 2018: 52) - dans le roman car ce sont bel et bien les aventures d'Esther (et non de Nejma) qui constituent le centre du récit. Quelque chose de similaire se joue dans Le Chercheur d'or et dans La Quarantaine, où une sorte de fusion imaginaire s'opère, à plusieurs reprises, entre Alexis et Ouma à Mananava, ou encore entre Léon et Suryavati sur l'île Plate. À chaque fois, la conscience du héros est comme fascinée, hantée, happée par l'existence de son âme-sœur, laquelle disparaît inopinément. Cependant, force est de constater que dans ces deux romans la voix masculine occupe tout le champ de la narration au détriment de celle du personnage féminin. En revanche un certain ré-équilibrage narratif a lieu dans Révolutions, un roman choral complexe qui déborde largement l'expérience singulière de Jean Marro.

Qu'en est-il dans Alma? On observe que ce roman est construit sur deux consciences jumelles, deux voix distinctes et deux parcours croisés. Jérémie Felsen fait résonner le "sanglot de l'homme blanc" dans le texte. ${ }^{3}$ Le retour au pays ancestral est lié au départ pour Jérémie à des recherches sur le dodo - l'oiseau fabuleux exterminé par les premiers colons européens de l'île Maurice - mais progressivement son enquête se transforme pour porter sur l'histoire de sa famille, sur les plantations sucrières et la question de l'esclavage. On retrouve dans ces pages la pulsion cartographique ${ }^{4}$ de l'auteur: en marge des sites enchanteurs, des plages, des clubs, des boîtes de nuit et des hôtels de luxe de l'île Maurice contemporaine, Jérémie dresse la carte détaillée des camps où étaient autrefois parqués les esclaves à travers l'île ainsi que des tours où les marrons étaient emprisonnés et châtiés. Le roman conjugue donc l'évocation, forcément fragmentaire, du passé colonial de Maurice à celle de son présent touristique. Le jour, Jérémie observe combien l'île est en train de changer, de se "moderniser" et se "développer" en accueillant les investissements et les galeries marchandes. Mais la nuit, tout semble être comme "avant", c'est-à-dire avant l'arrivée des Européens et l'exploitation des terres:

Je suis ici pour écouter la nuit au centre de l'île. [... ] Je me suis enveloppé dans ma parka imperméable, la tête posée sur mon sac, et j’ai regardé les étoiles apparaitre à travers la brume [... ]. C'est le même ciel que les marrons regardaient, nuit après nuit, dans l'angoisse de l'attente, guettant peut-être l'étoile qui les conduiraient à la Grande Terre, de l'autre côté de l'océan. [... ] Ici, à Macchabée, ils sont au milieu de l'océan, le ciel est nu, inchangé, introublé, sans rien qui menace ou pollue. Aucune lueur ici, aucune laitance. Seulement l'éclat des étoiles, les astres qui pulsent, qui les fixent, une puissance lointaine et familière. [...] Les esclaves fugitifs se souviennent de la nuit de leur enfance, ils marmonnent une incantation, une prière dans leur langue (Le Clézio 2017: 84-85).

\footnotetext{
2 Voir notamment Bruno Thibault, J.M.G. Le Clézio et la métaphore exotique (2009), en particulier le chapitre 8 intitulé "Étoile errante ou l'inorigine en partage".

3 Je renvoie ici à l'essai polémique de Pascal Bruckner, Le Sanglot de l'homme blanc: Tiers Monde, culpabilité, haine de soi (1983).

4 Sur ce point, voir Bruno Tritsmans 1990: 166.
} 
L'expérience de la fusion est ici remarquable car c'est d'abord aux esclaves marrons de l'île que songe Jérémie en contemplant le ciel nocturne. On note ici, d'une part, la fusion de ces deux points de vue, à la fois présent et passé, individuel et collectif. D’autre part ce passage marque la première évocation chamanique dans le texte, une incantation ou une prière adressée aux étoiles silencieuses. Les astres se tiennent haut dans le ciel noir d'encre, impassibles et inaccessibles, parés d'une aura brillante comme des divinités. Nous y reviendrons.

Doublant la voix des esclaves marrons d'antan, s'élève dans le roman la voix sourde, incohérente mais incessante de Dodo, le clochard qui hante les cimetières. Cette voix dit la misère, la solitude, l'errance et l'exclusion. Dodo, c'est l'homme réduit à sa plus simple expression, un être sans importance sociale, un corps rongé par la lèpre, une ombre qui s'efface, une voix que nul n'écoute, porteuse d'une mémoire chaotique et souffrante. Nous apprenons au fil des pages que Dodo est le fils d'Antoine Felsen marié à Rani Laroche, une créole de la Réunion aux cheveux crépus. Mais Antoine était lui-même le fils d'Achab Felsen marié à une femme indigène noire du Mozambique. Autrement dit, l'exclusion de Dodo fait résonner dans le texte, en contrepoint au récit de Jérémie, le non-dit colonial et raciste de l'île. Dodo est la pièce manquante du puzzle, le "chaînon" (Le Clézio 2017: 331) de l'arbre généalogique de la famille Felsen. Dodo a grandi dans une maison délabrée de l'autre côté du ruisseau et du bois de bambous qui marquaient la limite de la plantation d'Alma. L'enfant a été marginalisé par sa famille - comme l'avaient été son père et son grand-père avant lui. ${ }^{5}$ Dodo grandit seul, élevé par une nourrice noire, la vieille Yaya, "fille d'esclave" (ibid.: 63) qui lui raconte "zistoire Topsie" et "zistoire Sklavou" (ibid.: 64), les récits des esclaves prisonniers et des esclaves marrons. Arrivé à l'âge adulte, Dodo ne connaît pas un sort enviable: clochard et mendiant, il devient le souffre-douleur des voyous de Port-Louis.

\section{La rencontre de Jérémie et Dodo: une fusion ratée?}

Un jour, au seuil des années 80 , alors qu'il allait rendre visite à son oncle malade à Nice, Jérémie avait aidé un clochard tombé au milieu de la chaussée à se relever. "L'homme bredouillait des mots dans une langue étrange, mais ce qui m’a le plus surpris, c'est son visage sombre, sur lequel les traits semblaient avoir été effacés par une érosion ancienne, ou bien brûlés" (ibid.: 337). Jérémie avait traversé la chaussée en soutenant l'homme hébété, sous les coups de klaxon rageurs des automobilistes. Dodo et Jérémie se sont donc croisés l'espace d'un instant, non pas à Maurice mais en France, par hasard, quelques années avant le début du récit et le voyage de Jérémie sur l'île de ses ancêtres. Mais peut-on parler ici de rencontre ou de fusion? Les deux hommes n'échangent pas une seule parole, et ils ignorent tout l'un de l'autre.

5 Ironie suprême: Dodo a été écarté de la famille Felsen alors même que la ruine menaçait déjà cette famille. "L'expulsion d'Alma a déjà eu lieu [... et et de l'autre côté du rideau de bambous commencera la tragédie de Dominique, le fils de Rani dont le visage est rongé par la lèpre" (Le Clézio 2017: 333). 
C'est bien plus tard, au cours d'une conversation avec Emmeline Carcénac, une vague aïeule qui vit retirée à Maurice et qui a bien connu le père de Jérémie dans sa jeunesse, que celui-ci apprend l'existence de son cousin Dodo, sa marginalisation sociale sur l'île et sa disparition en France en 1982. À ce moment-là, la quête de Jérémie prend un nouveau sens: il abandonne son étude sur les oiseaux fossiles mais aussi son enquête sur les camps d'esclaves, les planteurs et les trafiquants. Il s'interroge à présent, de façon obsédante, sur "celui dont on ne parle plus, ce Fe'sen caché, un fantôme dans le placard, [... ] qui s'est perdu en France" (ibid.: 125).

Marianne Hirsch a développé la notion de postmémoire dans plusieurs essais au cours des années 90, notamment dans Family Frames (1997). La postmémoire décrit "la relation que la 'génération d'après' entretient avec le trauma culturel, collectif et personnel vécu par ceux qui l'ont précédée" (Hirsch 2014: 205-206). Il s'agit donc non de souvenirs personnels à proprement parler mais de souvenirs hérités et d'expériences "dont cette 'génération d'après' ne se 'souvient' que par le biais d'histoires, d'images et de comportements parmi lesquels elle a grandi” (ibid.: 206). Évidemment ces expériences transmises peuvent être violentes et lourdes d'affects car la souffrance des parents ou des aïeux se passent aux enfants. Le rapport de la postmémoire avec le passé est donc un mélange complexe de souvenirs rapportés, de douleurs ressenties chez les êtres chers, de projections et d'investissements imaginatifs, sur fond d'identification, d'horreur et de honte. Hirsch explique:

Grandir avec l'héritage d'écrasantes mémoires, être dominé par des récits qui ont précédé sa propre naissance ou sa propre conscience, fait courir le risque que les histoires de sa propre vie soient elles-mêmes déplacées, voire évacuées, par nos ascendants. C'est être formé, bien qu'indirectement, par des fragments traumatiques d'événements qui défient encore la reconstruction narrative et excèdent la compréhension. Ces événements sont survenus dans le passé, mais leurs effets continuent dans le présent (ibid.: 206).

Il est clair que la postmémoire n'est pas une position identitaire mais une structure de transmission générationnelle du traumatisme, le plus souvent par le biais de la famille. Mais, et cette question s'applique au roman de Le Clézio, si l'on adopte les expériences traumatiques d'autrui comme expériences à travers lesquelles on pourrait avoir vécu, si on les inscrit dans l'histoire de sa propre vie, peut-on pour autant le faire sans les imiter ou se les approprier indûment? Cette question fondamentale hante le récit d'Alma et modèle sa narration. Pour Dodo, c'est simple, le temps ne 'passe' pas car il vit plongé dans le temps du traumatisme, de l'abandon: "Je vis la même journée. Je ne sais pas comment c'est possible, mais c'est comme ça. [... C C'est que ma journée n'en finit pas, c'est une route sans fin, je ne vois pas la nuit arriver, je ne dors pas, et c'est le matin tout de suite” (Le Clézio 2017: 63). Pour Jérémie, en revanche, le processus d'identification, d'imagination et de projection est lié à son réseau familial et à l'héritage générationnel d'un secret honteux. Pour cette raison, Jérémie est animé par le besoin frustré de savoir quelque chose du traumatisme. Il est évident que la relation de ces deux personnages au passé traumatique n'est pas la même. C'est pourquoi la structure narrative d'Alma est double. Marine Landrot observe que les deux voix de Jérémie et de Dodo "bercent, martèlent, perforent" (ibid.: 36) le récit. "L’une provient d'un voyageur dans le temps, tour à tour intrépide et indolent, escaladant les branches de son arbre généalogique mauricien. L'autre sort de la bouche d'un vagabond lépreux, sans 
lèvres ni paupières, ne conjuguant ses verbes qu'au présent, même pour raconter ce qui fut et ce qui sera" (ibid.: 36). Ainsi, ajoute Landrot, les deux personnages incarnent dans Alma "les deux pôles magnétiques d'un même écrivain" (ibid.: 36). Dans ce roman, Le Clézio "se dédouble et se réincarne, se démultiplie et se recentre, pour creuser l'éternel sillon qui le mène de ses racines mauriciennes à l'harmonie céleste" (ibid.: 36). Mais, en réalité, les choses sont-elles si simples? Comme le souligne Isabelle Roussel-Gillet, "Alma n'est pas le roman à deux voix que l'on saisit à première vue” (2018: 187). D'autres voix s'ajoutent et se mêlent à celles des deux protagonistes: notamment celles de Saklavou, Topsie, Ashok, Artémisia et Marie-Madeleine Mahé qui forment "l'ethnofiction rhizomique" (ibid.: 187) de l'île. "Entre écoute et écriture, entre histoire et mémoire, entre trace et effacement" (ibid.: 188) se dessine un récit complexe, souvent poignant mais résolument incomplet car semé de silences et d'oublis plus ou moins volontaires. Ainsi, note Roussel-Gillet, le "roman polyphonique" (ibid.: 187) se transforme en "roman archéologique" (ibid.: 188): il cherche à déterrer les fantômes du passé. Au cours de ses vagabondages, Dodo erre dans les cimetières. Là, il aime suivre du doigt les lettres à demi effacées sur les tombes, et, tel un écrivain, les repasser à la craie ou au crayon...

Si Dodo incarne la structure de la mémoire traumatique, Jérémie incarne quant à lui la postmémoire dans le récit. Le jeune homme prend peu à peu conscience que le sens de son "pèlerinage" (Le Clézio 2017: 331) s'inscrit en marge de "l'histoire officielle" (ibid.: 331). Il prend aussi conscience que "l’histoire est un tissu en lambeaux" (ibid.: 319). Ayant retracé l'histoire secrète de Dodo, de Zaza et de Topsie, notamment grâce aux témoignages de Jeanne Tobie (ibid.: 93) et d’Emmeline Carcénac (ibid.: 100), Jérémie conclut:

Il y a toujours une part inachevée dans toute histoire, et celle que j'ai voulu reconstruire ne déroge pas à cette règle. [...] J'ai voulu recoller les morceaux d'une histoire brisée, celle des Felsen de l'île, à présent aussi éteints que l'oiseau lui-même, dead as a dodo. Peut-être était-ce de la vanité, ce sentiment d'appartenir à une tribu en train de disparaître, d'être le témoin, le signal faible et vacillant d'une autre ère, d'une autre culture [...] (ibid.: 335)?

Cette dernière phrase semble une confidence personnelle de l'auteur. Cependant, comme le souligne Bruno Jean-François, Dodo finit par s'éloigner de cette histoire familiale et insulaire dont Jérémie veut se rapprocher: "Ce jeu de trajectoires, à la fois inversées et en constant dialogue, est parfaitement représenté par le croisement des voix et des mémoires narratives. [... C'est sans doute dans cette intersubjectivité, dans cette errance dialogique et rhizomique de la narration qu'il importe de chercher l'histoire ou les histoires qu'explore l'auteur" (Jean-François 2019: 164).

Si Alma est bel et bien un roman du désenchantement, précisons cependant qu'il l'est à deux niveaux. D'une part l'écrivain note que l'expérience interculturelle y est inaboutie: "L’interculturel, une fois encore, n’aura pas lieu" (Le Clézio 2017: 221). D’autre part la postmémoire ne permet pas le retour à l'origine, à la source matricielle et fabuleuse: "C'est l'aube d'un temps ancien, elle allume l'horizon mais elle ne parvient pas à faire grandir le jour, c'est trop tard” (ibid.: 316).

${ }^{6}$ Le Clézio prend soin de raconter non seulement l'histoire de Topsie, l'esclave africain, mais aussi celle d'Ashok, le coolie indien. Ainsi sont représentés les deux grands groupes ethniques de l'île. 


\section{La rencontre avec le ciel: l'extase chamanique}

Nous avons parlé pour l'instant des personnages masculins qui dominent ce roman, Jérémie et Dodo. Mais il existe deux personnages féminins, certes secondaires, mais qui ne manquent pas d'intérêt, Krystal et Aditi. Ces deux jeunes femmes fascinent Jérémie: il ne cesse d'imaginer leur vie quotidienne, leurs sentiments, leurs pensées, leurs aspirations. À la tombée du jour, Krystal traîne dans les boîtes de nuit pour touristes ou dans les bars à prostituées. Délurée et délinquante, elle obsède Jérémie par son corps souple et séduisant. Aditi, quant à elle, travaille comme guide à la Mauritius Wildlife Foundation. Elle sillonne avec les touristes la "forêt endémique" (ibid.: 81) qui est l'ancien territoire des dodos et des esclaves marrons. Aditi habite "dans une cabane de bois" (ibid.: 134) à la lisière du parc national, avec d'autres jeunes militants écologistes venus d'Europe, d'Inde, d'Afrique du Sud et d'Australie. Pour autant, elle ne croit pas possible le retour à la nature. "C'est illusoire de vouloir conserver les choses comme si rien ne bougeait dans le monde", expliquet-elle à Jérémie, "et je n'aime pas cette idée de la nature vierge, il me semble parfois que c'est une idée de raciste, tu ne trouves pas?" (ibid.: 137). La Mauritius Wildlife Foundation n'est pour elle qu'un "prétexte pour vivre en forêt" (139) et se livrer à "son rituel, sa prière" (ibid.: 139). "Elle, c'est autre chose qu'elle vient chercher ici, quelque chose qui la relie au temps, au secret de la création, quelque chose d'aussi lointain et d'aussi constant que les chemins d'étoiles" (ibid.: 140).

En termes jungiens, si Dodo incarne dans le texte la figure ambiguë de l'ombre et du Soi, Krystal et Aditi incarnent en revanche la figure de l'anima initiatrice, également ambiguë. L'une (Krystal) est l'anima négative: séduite par l'illusion de la vie facile, puis victime des circonstances, elle aboutit finalement en prison. L'autre (Aditi) est l'anima positive, spirituelle et sidérale, qui mène l'initiation. ${ }^{7}$ C'est elle qui conduit Jérémie, un soir, à travers la forêt obscure vers "le centre du monde" (ibid.: 134). Le jeune homme y observe la jeune femme couchée sur une roche plate, au milieu des eaux, là même où deux torrents se rencontrent pour former la Rivière Noire. ${ }^{8}$ Dans un premier temps la peau d'Aditi, sombre et luisante, reflète la lueur des étoiles. Dans un second temps, son corps semble "confondu avec la roche" (ibid.: 134), ainsi qu'une divinité tellurique. Il est clair qu'Aditi est une figure fantasmatique et mythique, qui fait écho à d'autres figures de même acabit, telles Naja Naja dans Voyages de l'autre côté, Oya dans Onitsha, Ouma dans Le Chercheur d'or ou encore Suryavati dans La Quarantaine. ${ }^{9}$ En même temps, Aditi fait songer à l'artiste Ana Mendieta

\footnotetext{
7 Le Clézio note: "Aditi, cette jeune femme qui vit seule, est une sorte de combattante elle aussi, comme Krystal, mais dans un autre genre" (2017: 134). Dans la plus pure tradition leclézienne, Aditi accouche seule dans la forêt d'un enfant - fruit d'un viol, apprend-on à la page 192 -, tout en contemplant le ciel nocturne (ibid.: 274-76).

8 On reconnaît ici le décor du "Bout du monde" décrit dans Révolutions (pages 543-544). Catherine y accompagne son amie Somapraba qui l'initie à la spiritualité de l'Inde. Bien plus tard, Jean Marro explore ces lieux.

9 Précisons ici que le roman présente Aditi comme la descendante directe d'Ashok, l'homme qui a découvert Grand Bassin, le lac des fées situé au cœur de l'île Maurice, où l'on trouve aujourd'hui "tous ces temples et ces machins, et la statue géante de Shiva avec son trident" (Le Clézio 2017: 138).
} 
dont l'œuvre se situe à la croisée du Land art et du Body art. ${ }^{10}$ Immobile dans l'eau, Aditi psalmodie à voix basse les prières ancestrales qui rendent hommage à "ce qui existe dans ce lieu, dans la forêt, dans l'île, et qui n'appartient pas seulement à la mémoire des hommes" (ibid.: 140). Puis elle se tourne vers son compagnon: "Viens, dit-elle. Tu dois reconnaître le ciel" (ibid.: 141).

On voit ici que la muse de l'astronomie, liée au réalisme magique et à l'expérience chamanique, est bel et bien présente dans Alma. Sa présence s'affirme d'ailleurs de façon insistante à mesure que l'on se rapproche de la conclusion du récit. Il est clair que le ciel nocturne et ses astres mystérieux ne cessent de fasciner les deux narrateurs, Dodo et Jérémie. Cependant leur perspective n'est pas exactement la même. Examinons-les maintenant, l'une après l'autre.

Au cours de son voyage à pied à travers la France, de Paris à Nice, Dodo fait un bref séjour à l'Arche, une étrange communauté où vivent de jeunes bergers barbus et illuminés, menés par un gourou italien. Chaque soir, au moment de se coucher, les habitants de l'Arche contemplent longuement le ciel nocturne et le ballet des étoiles filantes:

[...] moi je reste les yeux ouverts devant l'entrée de la grotte et je compte les étoiles. [...] Même il y a une pluie d'étoiles, et le jeune homme barbu [...] dit: "C'est les Néréides". Je ne sais pas ce que c'est. Je demande: "Est-ce que les étoiles tombent sur la terre?’” Jonas rit un peu: "Non, non, elles sont très haut dans le ciel, elles brûlent avant de tomber". [... ] Je dis d'accord, et je vois le ciel qui tombe en petites lumières folles, les étoiles en train de mourir (ibid.: 298).

Après ce bref séjour à l'Arche, Dodo gagne la Côte d'Azur. Pour lui, Nice marque la fin du voyage. Il aboutit face à la mer, à bout de souffle et à bout de forces. Il est recueilli à la Maison Blanche, "la maison des indigents et des aliénés" (ibid.: 323), située dans un grand jardin en marge de la ville. "Ici, à cause des aliénés, il n'y a pas de miroirs où se cachent les démons" (ibid.: 325), observe Dodo, et "les fenêtres sont bouchées par des grilles noires" (ibid.: 323). Au cours d'une scène qui n'est pas sans faire écho aux pages finales du ProcèsVerbal, un médecin psychiatre vient examiner Dodo. Le médecin est entouré d'étudiants vêtus de blouses blanches. Parmi eux, une jeune femme, nommée Aïcha, s'intéresse aux aventures de Dodo et engage le dialogue avec lui. Le clochard évoque confusément sa vie à Maurice, son arrivée récente en France, son épuisement physique et moral. "Ici, à la Maison Blanche, pe'sonne ne me connait, je suis vraiment pe'sonne. Je ne veux plus aller nulle part ailleurs" (ibid.: 323), dit-il. Puis, comme Adam Pollo dans Le Procès-Verbal, Dodo s'installe à l'écart et s'enfonce dans le mutisme: "Je reste immobile dans le soleil d'or, les yeux levés vers l'intérieur de ma tête puisque je ne peux pas dormir, un jour mon âme va partir par un trou dans ma tête, pour aller au ciel où sont les étoiles" (ibid.: 328).

Cette image est la dernière manifestation du personnage dans le texte. On note dans cette citation le jeu symbolique de la lumière (le soleil) et de la nuit obscure (les étoiles) ainsi que le passage non moins symbolique de l'aveuglement à la vision: Dodo, les yeux révulsés, imagine son âme s'envoler par un trou dans sa tête, exactement comme dans la transe chamanique. C'est un mouvement à la fois d'introversion radicale ("les yeux vers

10 Ana Mendieta (1948-1985) est une performeuse, sculptrice, peintre, photographe et artiste vidéo américano-cubaine dont l'œuvre se caractérise par le lien earth-body et le chamanisme santéria. Plusieurs œuvres photographiques de Mendieta la présentent allongée nue dans un cours d'eau. 
l'intérieur de ma tête") et d'extase cosmique ("aller au ciel où sont les étoiles"). On note aussi le dénuement absolu de Dodo à l'asile qui n'est pas sans évoquer celui des mendiants mystiques des légendes chrétiennes ou hindoues. En même temps on observe que cette thématique mystique est présentée sous une forme à la fois très allusive et très ambiguë. Dodo est un homme au bout du rouleau, en fin de vie, qui perd la tête. Il attend la mort et il a compris, depuis son séjour à l'Arche, que les étoiles elles-mêmes sont "en train de mourir".

Qu'en est-il de Jérémie? En arrivant à Maurice, Jérémie se laisse d’abord séduire par la beauté tropicale de l'île, ses palmiers, ses montagnes et ses lagons, et surtout par "le coucher de soleil chromo" (ibid.: 60) qui fait rêver les touristes. Ce coucher de soleil chromo, on le retrouve à la page 336, sous la forme d'affiches publicitaires dans l'agence de voyage tenue par un vague cousin de Jérémie, Jackie Marzen: “[... ] des photos chromos qui vantent les merveilles du coucher de soleil sur le lagon ou de la pêche en gros” (ibid.: 336). Cet encadrement malicieux du récit par une image trompeuse de Maurice ne saurait pourtant dissimuler l'envers du décor: le règne de l'argent, les expropriations, l'exploitation touristique et le développement anarchique de l'île. Parvenu à la fin de son séjour à l'île Maurice, Jérémie se tourne lui aussi face au ciel:

Le ciel est devant moi, face au sud chaque nuit, mais je ne l'ai jamais regardé si longtemps, peut-être parce que je m'en vais, et que je veux imprimer chaque signe, chaque figure sur mes rétines. [...] C'est le zénith que j'emporte, le point aveugle vers lequel tout converge, et est-ce par hasard s'il est entouré de tous ceux que j'aime, Grus, Columba, Phœnix, Corvus, et l'oiseau sans nom qui trace une croix de son corps et de ses ailes, dardé vers le sud absolu? (ibid.: 318).

Contrairement à Dodo, Jérémie a toute sa vie devant lui et il a "des étoiles plein les yeux". Le ciel nocturne est pour lui un espace mythique dans lequel les constellations figurent des oiseaux de légende. Le réseau sémantique de l'extase chamanique est ici encore très marqué: le "zénith" et le "point aveugle" vers lesquels tout semble converger - comme en une sorte de vortex vertical - renvoient à une vision cosmique indicible. On trouve donc dans ce roman à la fois une certaine exaltation (Jérémie) et une certaine déflation (Dodo) de la vision mythique, laquelle est associée aux constellations visibles dans le ciel nocturne. Cette vision mythique constitue-t-elle pour l'écrivain une échappatoire, une façon de contrer la hantise de la mémoire en se projetant vers une origine absolue, idéale, inaccessible? En tout cas, Alma apparaît comme l'un de ces "récits indécidables" dont la fiction, souligne Bruno Blanckeman, "est distancée ou contestée en son for, par un usage ambivalent de ses paramètres", et dont l'intrigue "se décale, se dédouble, se défait" (2000: 16). Une certaine "schize" gît au cœur du récit car l'écrivain "produit de la fiction et surligne cette production, énonce du romanesque et le dénonce comme tel" (ibid.: 17). Mais le réalisme magique ne réside-t-il pas précisément, tout entier, dans cette schize de la vision et de la narration dédoublée? 


\section{Bibliographie}

Blanckeman, Bruno 2000. Les Récits indécidables: Jean Echenoz, Hervé Guibert, Pascal Quignard. Villeneuve d'Ascq: Presses Universitaires du Septentrion.

Bruckner, Pascal 1983. Le Sanglot de l'homme blanc: Tiers Monde, culpabilité, haine de soi. Paris: Seuil / L'Histoire immédiate.

Hirsch, Marianne 1997. Family Frames: Photography, Narrative, and Postmemory. Cambridge: Harvard University Press.

— 2014. "Postmémoire”. Traduit par Philippe Mesnard. Témoigner: entre histoire et mémoire 118 : 205-206.

Imbert, Jean-Philippe 1997. “J.M.G. Le Clézio, Writer of Exile in Désert and Étoile errante”. In: Anthony Coulson (ed.). Exiles and Migrants: Crossings Thresholds in European Society. Brighton: Sussex Academic Press.

Jean-François, Emmanuel Bruno 2019. "Mémoire, corps et errance dans Alma de J.M.G. Le Clézio”. In: Justine Feyereisen [\&] Paul Dirkx (dirs.). Corps. Les Cahiers J.M.G. Le Clézio 12: $157-182$.

Kelly, Van 2018. "Mapping Self and/as Other: Attempts to Fuse Horizons in Modiano and Le Clézio". Yale French Studies 133 (janvier): 39-57.

Landrot, Marine 2017. “Alma de J.M.G. Le Clézio”. Télérama 3534 (3 octobre 2017): 36.

Le Clézio, Jean-Marie Gustave 2017. Alma. Paris: Gallimard.

1992. Étoile errante. Paris: Gallimard.

Putnam, Walter 1998. “The Poetics and Politics of Space in J.M.G. Le Clézio's Étoile errante”. In: E. Barkan et M.-D. Shelton (eds.). Borders, Exiles, Diasporas. Stanford: Stanford University Press.

Roussel-Gillet, Isabelle 2018. “Alma (2017) de J.M.G. Le Clézio”. In: Sabrinelle Bedrane [\&] Sophie Jollin-Bertocchi (dirs.). Romanesques, Romances. Les Cahiers J.M.G. Le Clézio 11: 187-191. Passage(s).

Thibault, Bruno 2009. J.M.G. Le Clézio et la métaphore exotique. Amsterdam \& New York: Éditions Rodopi.

Tritsmans, Bruno 1990. “Rêves de cartes: Récit et géométrie chez Gracq et Le Clézio”. Poétique 82 (avril): 165-177. 